\title{
Doctor, no tengo cita. ¿Podría atenderme?
}

\author{
Raquel González Carnero ${ }^{a}$, Lara Luna del Pozo ${ }^{\mathrm{b}}$, Ana Isabel Rabadán Velasco ${ }^{\mathrm{b}}, \mathrm{M}^{\mathrm{a}}$ Dolores \\ Martínez Malabiac y Francisco Javier Alonso Moreno ${ }^{d}$
}

Recibido el 2 de febrero de 2012.

Aceptado para su publicación el 10 de septiembre de 2012.

\begin{abstract}
RESUMEN
Un paciente sin cita es algo muy frecuente en Atención Primaria (AP) y puede llegar a ser problemático. Estos pacientes ponen a prueba al Médico de Familia, ya que ha de saber valorar si la consulta no debe demorarse, a la vez que ha de hacer educación sanitaria, para que los pacientes sigan los cauces habituales para la demanda de atención. Invitamos a la reflexión y mostramos que no por ser una consulta de última hora tiene menos importancia, por lo que hemos de dedicarle el tiempo necesario para llevar a cabo una entrevista clínica correcta y hacer un diagnóstico diferencial, descartando patologías de gran importancia que pudiera presentar nuestro último paciente de la mañana.
\end{abstract}

Palabras clave: Atención Primaria de Salud, Relaciones Profesional-Paciente, Embolia Pulmonar.

\section{ABSTRACT}

Title: Doctor, I don't have an appointment. Can you see me?

A patient without an appointment is quite a common occurrence in Primary Health care, and can be a problem. These patients test the family doctor, as he/she has to be able to judge whether the appointment should, or should not, be delayed, while at the same time giving healthcare education, so that the patients learn to follow the usual channels to obtain care. We invite reflection and show that in spite of being a last-minute consultation, it is no less important, which is why we should dedicate the necessary time to carry out a proper clinical interview and make a differential diagnosis, ruling out any important pathologies which our last patient of the morning could be suffering from.

Key words: Primary Health Care, Proffesional-Patient Relations, Pulmonay Embollsm.

\section{INTRODUCCIÓN}

La atención urgente o sin cita dentro del horario habitual de consulta de los Equipos de Atención Primaria plantea a menudo problemas de organización del centro y de la consulta ${ }^{1}$. Trastorna el ritmo de las consultas y produce malestar en el resto de pacientes, que pueden percibir como inferior la atención que reciben de su médico junto con la demora en su espera, a menudo no justificada, dado que las patologías atendidas no siempre podrían considerarse urgencias objetivas².

Las urgencias se clasifican en: subjetivas, cuando se percibe una alteración de la salud que provoca la solicitud de una respuesta rápida del sistema; y objetivas, cuando el médico considera necesaria una atención inmediata ${ }^{1,2}$. Existen diversos motivos por los que los pacientes acuden sin cita previa a la consulta, como la urgencia médica, acudir como acompañante y a por recetas. Algunos de estos motivos se pueden relacionar con variables como encontrar el teléfono ocupado, la inactividad laboral si se acude como acompañante, el desconocimiento del sistema en el caso de pacientes extranjeros y la incompatibilidad de horario laboral en personas de nivel de estudios alto ${ }^{3}$. En otros casos, acudir sin cita previa puede deberse a haber recibido el alta hospitalaria y el paciente acude a por las recetas prescritas que no han sido facilitadas.

Por todo ello, decidimos redactar el caso de uno de nuestros pacientes que nos pidió ser atendido a pesar de no tener cita, ya que acudió por un motivo urgente que debíamos valorar. 


\section{OBSERVACIONES CLÍNICAS}

Se trata de un varón de 28 años, que acude sin cita a última hora, con dolor en hemitórax derecho, iniciado de madrugada, con carácter pleurítico y sin irradiación. Es fumador de diez cigarrillos diarios, con antecedente de asma extrínseca estacional, abdominoplastia hace años e intervención por colesteatoma en oído izquierdo. Hace vida sedentaria. No refería proceso catarral previo, aunque sí cierta sensación de falta de aire.

La auscultación cardio-pulmonar fue normal. Saturación de oxígeno $97 \%$ y frecuencia cardiaca de $75 \mathrm{lpm}$. El paciente fue derivado al Servicio de Urgencias para estudio radiológico y valoración, con sospecha de neumotórax.

En Urgencias hospitalarias, el paciente refería además disnea de pequeños esfuerzos y que en los días previos presentó dolor escapular que no nos había comentado a nosotros. La exploración era normal.Serealizó un electrocardiograma, radiografía de tórax y hemograma, con resultado normal. En la bioquímica sólo destacaba elevación del D-dímero de $2.594 \mathrm{ng} / \mathrm{ml}$. Por este motivo se realizó un TAC torácico, que mostró tromboembolismo pulmonar (TEP) bilateral, por lo que ingresa en Neumología. Reinterrogando al paciente, comentó que había presentado una lesión deportiva en el miembro inferior (MI) derecho, que se había vendado, con signo de Homans negativo. Durante el ingreso se hizo un estudio con ecodoppler de miembros inferiores, en el que se objetivó un trombo en vena poplítea de MI izquierdo de aspecto reciente. MI derecho sin alteraciones. Se realizó un estudio de trombofilia, cuyo resultado ha sido normal.

\section{COMENTARIOS}

El dolor torácico es un motivo frecuente de consulta médica, que puede estar originado tanto por patologías banales como por verdaderas emergencias médicas. Debido a que suele demandar atención médica inmediata, es fundamental realizar una completa anamnesis y una detallada exploración física, para diferenciar si su origen es respiratorio, cardiológico u osteomuscular. Las causas de dolor torácico ${ }^{4}$ de origen cardiovascular se presentan en la tabla 1.

\begin{tabular}{l|l|l}
\hline \multicolumn{1}{c|}{ Miocárdico } & \multicolumn{1}{c|}{ Pericárdico } & \multicolumn{1}{c}{ Grandes vasos } \\
\hline Ángor & Pericarditis & Disección aórtica \\
Infarto & Taponamiento & Tromboembolismo pulmonar \\
Valvulopatía aórtica & Tumor & Infarto pulmonar \\
Miocarditis hipertrófica & & Hipertensión pulmonar \\
Rotura de cuerda tendinosa & & HTA sistémica severa \\
Aneurisma seno de Valsalva & & Sobrecarga ventrículo derecho \\
Hipertiroidismo & & \\
Anemia severa & & \\
Policitemia & & \\
Ejercicio intenso & & \\
\hline
\end{tabular}

Tabla 1. Causas de dolor torácico de origen cardiovascular.

En cursiva: causas muy graves, que precisan derivación obligatoria al Servicio de Urgencias Hospitalario.

Modificado de: Real MA, Fernández ME, Imaz M, Calvo E, Abreu MA. Atención a urgencias en un centro de salud. En: Gil VF, Merino J, Orozco D, Quirce F. Manual de Metodología de Trabajo en Atención Primaria. Madrid: Jarpyo Editores;1997.p.1380. 
EI TEP es un proceso patológico frecuente, con una elevada tasa de casos no diagnosticados, a pesar de que en la literatura médica actual se dispone de abundante información sobre métodos diagnósticos y guías clínicas. Se puede presentar como un cuadro poco definido y no siempre de forma aguda. Es importante pensar en él para el diagnóstico diferencial en Atención Primaria ya que, en general, es reversible, total o parcialmente, con el tratamiento adecuado, y por las graves consecuencias que acarrearía no tratarlo ${ }^{5}$.

Las trombosis venosas que se producen en el sistema venoso profundo son más importantes que las del sistema venoso superficial, debido a la gravedad de sus complicaciones potenciales (embolia pulmonar, síndrome postrombótico). Los trombos localizados a nivel poplíteo o en áreas más proximales presentan mayor riesgo de producir embolia pulmonar; un $50 \%$ de las trombosis venosas profundas (TVP) proximales embolizarán, frente a un $30 \%$ de las distales ${ }^{6}$.

Se debe considerar el diagnóstico de TEP ante la presencia de los 3 signos y síntomas más frecuentes: disnea, dolor, pleurítico y taquipnea ${ }^{7}$ .Otros signos y síntomas que puede desarrollar el TEP son: tos, hemoptisis, presíncope, fiebre, diaforesis profusa, crepitantes, taquicardia, refuerzo del $2^{\circ}$ tono, presencia de $4^{\circ}$ tono, roce pleural, cianosis, etc. Se han diseñado modelos predictivos estandarizados que clasifican a los pacientes en baja, media y alta probabilidad, como el de Wells et $\left.a\right|^{8}$. (Tabla 2).

\begin{tabular}{l|c}
\hline Variables & Puntos * $^{*}$ \\
\hline TEP es más probable que un diagnóstico alternativo & 3 \\
Signos y síntomas de TVP (edema, dolor a la palpación de venas profundas) & 3 \\
Historia previa de enfermedad tromboembólica venosa (TVP/TEP) & 1,5 \\
Cirugía o inmovilización en el último mes & 1,5 \\
Frecuencia cardiaca $>100$ latidos/min. & 1 \\
Hemoptisis & 1 \\
Cáncer activo (tratamiento continuo o en los 6 meses previos o paliativo) & 1,5 \\
\hline
\end{tabular}

Tabla 2. Escala para la probabilidad clínica de TEP de Wells et al.

* Puntuación: <2 puntos indica una baja probabilidad (probabilidad TEP 3,4\%); 2 a 6 puntos, probabilidad moderada (probabilidad TEP 27,8\%) y $>6$ puntos, una alta probabilidad de TEP (probabilidad de TEP 78,4\%).

TEP: tromboembolia pulmonar; TVP: Trombosis Venosa Profunda.

Fuente: Wells PD. Use of a clinical model for safe management of patients with suspected pulmonary embolism.

Ann Intern Med. 1998;129:997-1005.

La enfermedad tromboembólica venosa (ETV) es un problema de salud importante, con una incidencia anual de 1-2 casos por cada 1.000 personas/año. Uno de cada tres pacientes con ETV sintomática presenta embolia pulmonar, que es a menudo infradiagnosticada por su presentación clínica, variable e inespecífica. Por lo tanto, esta posibilidad diagnóstica, no sólo se debe considerarse para los pacientes con síntomas agudos, sino también para las personas con síntomas respiratorios ${ }^{9}$.

El mejor tratamiento de la enfermedad tromboembólica venosa, que incluye la TVP y el TEP, es la prevención. En el caso del tratamiento con anticoagulantes del TEP supone prevención secundaria de las complicaciones ${ }^{5}$.
Una vez diagnosticado el TEP, los principales objetivos a corto plazo son la prevención de la extensión del trombo, la embolización pulmonar y la aparición de embolismos recurrentes, por lo que se recomienda prontitud en la instauración del tratamiento. A largo plazo, el tratamiento pretende evitar recidivas tardías y secuelas, como el síndrome posflebítico y la hipertensión pulmonar. Habitualmente, la elección de la pauta se hace en función de la gravedad y la forma de presentación de la enfermedad, mientras que la presencia y el tipo de factores de riesgo condicionan la decisión sobre su intensidad y duración ${ }^{5}$. 
En la mayoría de los pacientes, la terapéutica anticoagulante aislada es suficiente para conseguir los objetivos: ante la sospecha de TEP, y después de descartar contraindicaciones para la anticoagulación, se administra tratamiento con heparina de bajo peso molecular (HBPM) en dosis variables según el peso del paciente. Simultáneamente, se inicia tratamiento con anticoagulantes orales (ACO), ajustando las dosis para mantener una razón normalizada internacional entre 2 y 3 . El tratamiento con HBPM se mantiene durante un mínimo de 5 días y sólo se suspende cuando los ACO han alcanzado un nivel terapéutico durante 2 días consecutivos. En el caso de embolismo pulmonar masivo o submasivo, así como en pacientes que presentan una trombosis ileofemoral grave, conviene alargar el tratamiento con HBPM durante 7-10 días. La duración del tratamiento anticoagulante es variable según el caso: en un primer episodio y con factores de riesgo transitorio, el tratamiento se mantendría 3 meses o hasta que desaparezcan dichos factores; si los factores de riesgo son continuos, el tratamiento debería mantenerse de manera indefinida. En un primer episodio de ETV de origen idiopático o en sujetos con trombofilia, el tratamiento tendría una duración mínima de 6-12 meses, aunque se postula mantener un tratamiento indefinido en caso de trombofilia y estados de alteraciones primarias de la coagulación. En caso de 2 o más episodios de ETV, el tratamiento anticoagulante debe mantenerse de manera indefinida ${ }^{5}$. En la tabla 3 se revisa la duración del tratamiento anticoagulante en pacientes con ETV.

\begin{tabular}{lc}
\hline Situaciones clínicas & Tiempo \\
\hline $\begin{array}{l}\text { TVP asociada a un factor de riesgo transitorio (traumatismo, cirugía, inmovilidad, terapia } \\
\text { estrogénica,...) }\end{array}$ & 3 meses \\
Primer episodio de TVP idiopática (sin factor de riesgo reconocible) & 6 meses \\
$\begin{array}{l}\text { Presencia de factor de riesgo permanente o hereditario (malignidad, Homocigotos Factor V Leiden, } \\
\text { Anticuerpos antifosfolípido, Deficiencia de Antitrombina III, Proteína C, o Proteína S) }\end{array}$ & Indefinida \\
TVP recurrente (dos o más episodios) & Indefinida \\
\hline
\end{tabular}

Tabla 3. Duración del tratamiento anticoagulante en pacientes con enfermedad tromboembólica venosa.

TVP: Trombosis Venosa Profunda.

En caso de que los anticoagulantes orales estuvieran contraindicados (embarazo, alergia...) o fuesen inefectivos (por ejemplo, algunos pacientes con cáncer) la terapia de mantenimiento se realizará con heparina subcutánea.

Fuente: Villa Estébanez R, Veiras del Río O. Los principales problemas de salud. Trombosis venosa profunda. AMF 2009;5(1):11-20.

\section{BIBLIOGRAFÍA}

1. Grupo de Trabajo de la Sociedad Andaluza de Medicina Familiar y Comunitaria (SAMFYC). Ordenación de las urgencias en atención primaria. Aten Primaria. 1992;9:269275.

2. Vítores Picón MP, Cortés Durán PM, Fernández Girón M, Torres Romo L, Tomey Torcal I, Iradier Barrio D et al. Evaluación de la organización en la atención a pacientes sin cita. Aten Primaria. 2001;28:298-304.

3. De Dios del Valle R, Franco Vidal A, Tena Ortega G, Muedra Sánchez M, Hernández Sánchez A, Grueso Muñoz E. ¿Por qué hay pacientes que acuden sin cita previa? SEMERGEN. 2006;32(1):4-9.

4. Real MA, Fernández ME, Imaz M, Calvo E, Abreu MA. Atención a urgencias en un centro de salud. En: Gil VF, Merino J, Orozco D, Quirce F. Manual de Metodología de Trabajo en Atención Primaria. Madrid: Jarpyo Editores; 1997.p.1380.
5. Lloveras Clos MM, Corominas Alunes C, Servent Batlle C y Rodríguez Jerez F. ¿Neumonía o tromboembolismo pulmonar? A propósito de un caso. FMC. 2007;14(2):83-6.

6. Villa Estébanez R, Veiras del Río O. Los principales problemas de salud. Trombosis venosa profunda. AMF. 2009;5(1):11-20.

7. Uresandi F, Blanquer J, Conget F, Gregorio MA, Lobo JL, Otero R, et al. Guía para el diagnóstico, tratamiento y seguimiento de la tromboembolia pulmonar. Arch Bronconeumol. 2004;40:580-94.

8. Wells PS, Anderson DR, Rodger M, Ginsberg JS, Kearon $C$, Gent $M$ et al. Derivation of a simple clinical model to categorize patients probability of pulmonary embolism: increasing the models utility with the SimpliRED D-dimer. Thromb Haemost. 2000;83(3):416-20.

9. García-Sanz MT, Peña-Álvarez C, González-Barcala FJ. Trombo-embolismo pulmonar: necesitamos sospecha clínica. An Sist Sanit Navar. 2012;35(1):115-20. 